\title{
Impact of Distributed-Memory Parallel Processing Approach on Performance Enhancing of Multicomputer-Multicore Systems: A Review
}

\section{Dildar Masood Abdulqader}

Department of Information Technology, Akre Technical College, Duhok Polytechnic University, Duhok, Kurdistan Region, Iraq

dildar.masood@dpu.edu.krd

\section{Subhi R. M. Zeebaree}

Culture Centre, Duhok Polytechnic University, Duhok, Kurdistan Region, Iraq. subhi.rafeeq@dpu.edu.krd

\section{ARTICLE INFO}

\section{Article History:}

Received: 2/10/2021

Accepted: 16/11/2021

Published: Autumn 2021

\section{Keywords:}

Multicomputer, multicore, distributed system, parallel processing, performance enhancing.

Doi:

10.25212/lfu.qzj.6.4.45

\section{ABSTRACT}

Distributed memory is a term used in computer science to describe a multiprocessor computer system in which each processor has its own private memory. Computational jobs can only work with local data, so if you need remote data, you'll have to communicate with one or more remote processors. Parallel and distributed computing are frequently used together. Distributed parallel computing employs many computing devices to process tasks in parallel, whereas parallel computing on a single computer uses multiple processors to execute tasks in parallel. Distributed systems are designed separately from the core network. There are different kinds of distributed systems such as peer-to-peer (P2P) networks, groups, grids, distributed storage systems. The multicore processor can be classified into two types: homogeneous and heterogeneous. This paper reviews the impact of the distributed-memory parallel processing approach on performance-enhancing of multicomputer- 


\section{QALAAI ZANISTSCIENTIFIC JOURNAL}

A Scientific Quarterly Refereed Journal Issued by Lebanese French University - Erbil, Kurdistan, Iraq

Vol. (6), No (4), Autumn 2021

ISSN 2518-6566 (Online) - ISSN 2518-6558 (Print)

multicore systems. Also, number of methods have been introduced which used in distributed-memory systems and discuss which method is the best and enhance multicore performance in distributed systems. The best methods were those which used an operating system named gun/Linux 4.8.0-36, intel Xeon 2.5, python programming language.

\section{Introduction}

A distributed system is a group of standalone computers that appear to their users as one coherent system. Distributed computing uses many remote computers geographically and solves large and complex tasks with high efficiency (Agarwal 2004)(Dino et al. n.d.). However, when deployed through distributor applications today in data centres, it is possible to participate in the design of distributed systems with their own network layer, but can offer significant benefits (Ports et al. 2015). Distributed systems display the best price and performance than concentrate systems(Van Steen and Tanenbaum 2017). Computer power can be added in a tiny boost to distributed systems. Distributed systems allow multiple users to access a shared computing resource, providing resource sharing (Bansal, Sharma, and Trivedi 2011)(Shukur et al. 2020).

Multicore processors have become popular and have a compact parallel computing power that cannot be completely used unless the program in progress is written accordingly. Writing an effective, scalable parallel program is very complex. In order to get the most out of multi-core processors, many parallels are needed in order to effective implementation of a program on a higher number of cores effectively. Simultaneous execution of several programs on several cores (Bridges et al. 2008) (McCool 2008). Multi-core processors have been around since the last decade, but they gained importance later on due to the limitations of technology that single-core processors face today(Wang et al. 2010) such as high throughput and long battery life with high energy efficiency(Ramanathan 2006). Multicore processors can be 
Vol. (6), No (4), Autumn 2021

ISSN 2518-6566 (Online) - ISSN 2518-6558 (Print)

implemented in many ways depending on the application requirements. It can be implemented either as a heterogeneous group, as a homogenous group and a mixture of the two(Blake, Dreslinski, and Mudge 2009).

This paper introduces the performance-enhancing of multicomputer-multicore distributed memory systems based on parallel processing principles in section 2 and section 3. Section 4 gives a literature review about performance-enhancing of multicomputer-multicore distributed memory systems based on parallel processing principles and discussion of the surveyed methods. Section 5 is the conclusion of the review paper.

\section{Distributed system}

Distributed systems are designed separately from the core network, making the worst assumptions (Ports et al. 2015). Furthermore, the issue of operating systems display in distributed systems proposes new solutions to existing problems (Lu et al. 2016) (Haji et al. 2020). Distributed systems allow multiple users to access a shared computing resource, providing resource sharing. Examples of distributed computing include online rail reservation systems, air traffic control, internet banking, etc.(Bansal et al. 2011).

\section{a. Peer-to-Peer (P2P) Distributed Systems}

P2P is a class of application that benefits from the storage of resources, courses, content, and human existence that are accessible on the Internet frontier. Since recovering these decentralized resources means working in an unstable connections environment and unpredicted IP addresses, P2P nodes need to work outside of DNS and have worthy or complete independence for intermediate servers(Wang and $\mathrm{Li}$ 2003).

The existing Peer-to-Peer (P2P) networks can be categorized into three generations: First generation: The first P2P networks, such as napster and gnutella, aim to spread easily and quickly. Second generation: P2P networks typically use DHT technology to achieve better scalability and better query efficiency, and provide load balancing and 


\section{QALAAI ZANISTSCIENTIFIC JOURNAL \\ A Scientific Quarterly Refereed Journal Issued by Lebanese French University - Erbil, Kurdistan, Iraq}

Vol. (6), No (4), Autumn 2021

ISSN 2518-6566 (Online) - ISSN 2518-6558 (Print)

inevitable search guarantees. Third generation: The recently proposed P2P network aims to provide high flexibility assuming that the node will collapse with some possibility of failure(Fiat and Saia 2002). The structure means that the topology of the P2P network is tightly controlled (such as Mesh(Zhao, Kubiatowicz, and Joseph 2001) (Rowstron and Druschel 2001), Ring(Stoica et al. 2001), d-dimension Torus(Ratnasamy et al. 2001)).

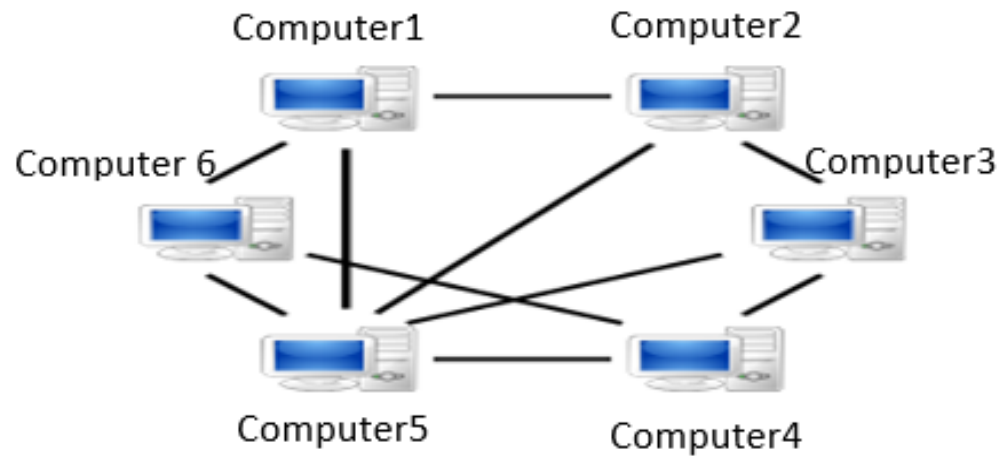

Figure (1): P2P (Peer-to-Peer) distributed systems (Anton and Norbert 2016).

\section{b. Clusters Distributed Systems}

A computer group is a group of interconnected computers that are habitually linked using a fast local area network (LAN). Computer clusters are the preferred distributed system construction type because of the top performance-price divided computing ratio. It makes up the rest are Massively Parallel Processors (MPP) and Pleiades systems (Grudenić and Bogunović 2009). It's a local area network with high-speed connections and more common in modern high-performance computing.

Number of computers are grouped so that benefiting from an individual resource group. Then, the result of the minimum job will be merged to form the end outcome (Amir et al. 2004). Cluster computing aid organizations raise their computing power by apply generally available standard technology. These hardware and software, known as commodities, can be bought on the market at a comparatively small 


\section{QALAAI ZANISTSCIENTIFIC JOURNAL \\ A Scientific Quarterly Refereed Journal Issued by Lebanese French University - Erbil, Kurdistan, Iraq}

Vol. (6), No (4), Autumn 2021

ISSN 2518-6566 (Online) - ISSN 2518-6558 (Print)

cost(Lakshmanan, Ahamad, and Venkateswaran 2003). A set of computers combination together in such a way that a single resource group makes a group. Then, the result of the minimum job will be merged to form the end outcome(Amir et al. 2004).

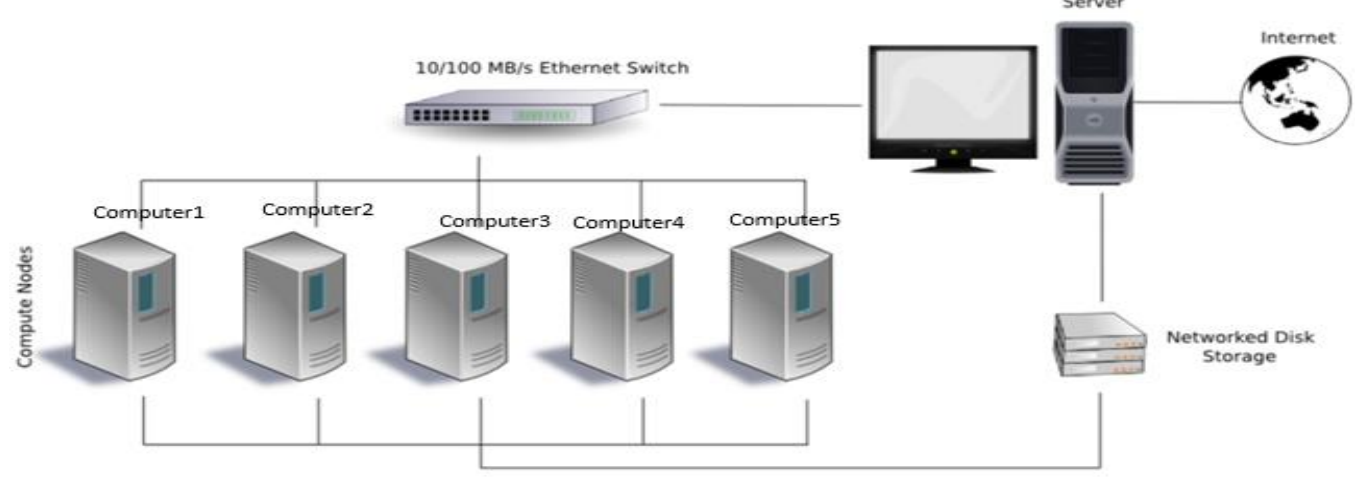

Figure (2): Clusters distributed systems (Sweilam, Moharram, and Ahmed 2012).

\section{c. Grids Distributed Systems}

Grid computing is a group of computational resources from a variety of administrative areas used for a usual task, technical, or commercial problem that requires a large number of computer training courses or the need for large amounts of data processing. It is a kind of parallel distributed system that allows you to dynamically distribute, select, and edit independent geographically distributed sources over time, depending on the availability, power, performance, cost, and quality of service requirements for users (Saafan 2009)(Rashid et al. 2018).

The broker used in Network Transactions is responsible for assigning tasks. The size of a transition system can vary from a few hundred computers in a large organization to thousands of nodes in many organizations. Smaller networks that are confined to one organization are commonly known as a company within a node, while a larger system is referred to as a joint node (Puttaswamy, Zheng, and Zhao 2008). 


\section{QALAAI ZANISTSCIENTIFIC JOURNAL}

A Scientific Quarterly Refereed Journal Issued by Lebanese French University - Erbil, Kurdistan, Iraq

Vol. (6), No (4), Autumn 2021

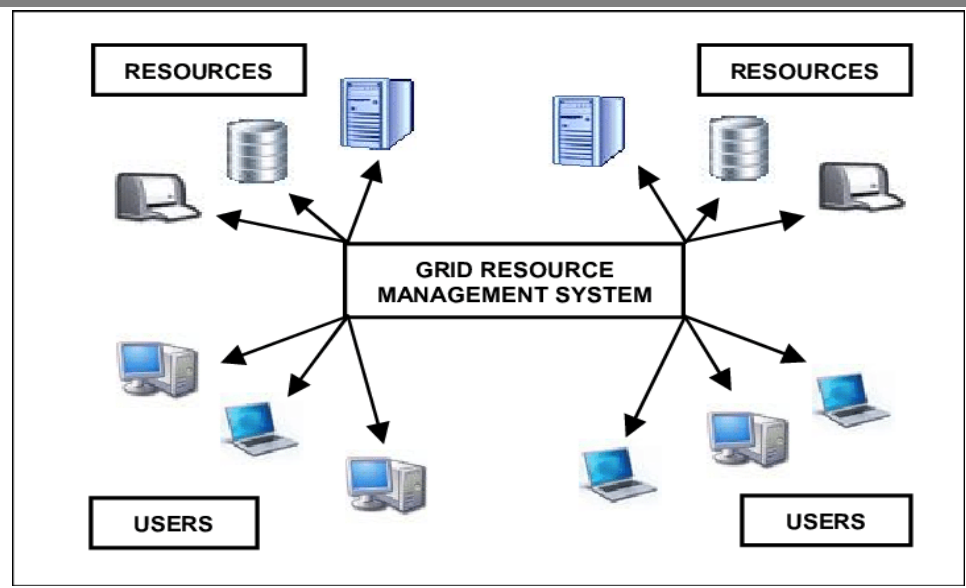

Figure (3): Grids distributed systems (Kaur, Kaur, and Kaur 2013).

\section{d. Distributed Storage Systems}

The rapid growth of storage capacity, account resources, and bandwidth combined with the lower cost of storage equipment has increased the popularity of distributed storage systems(Harinath et al. 2015). The main kind of distributed storage systems. In particular, redundant array of inexpensive disks (RAID), central raid, network storage, and Local Area network (LAN). Network-attached storage NAS and storage area network SAN are the four most common distributed storage technologies.

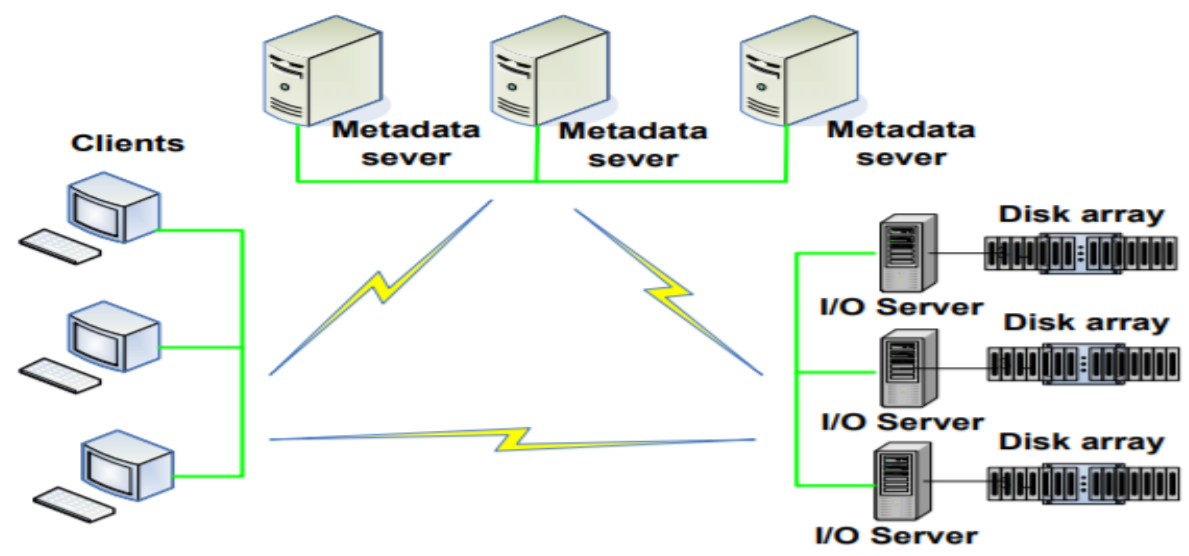

Figure (4): Distributed storage systems (Chen et al. 2011). 


\section{Multicore}

Usually, a multi-core processor is a single processor that has several cores on a chip(Wang et al. 2010). This is due to the presence of parallel processing technology that was absent in a single-core processor. There are CPUs with different basic levels two-cores, four-cores, six-cores, eight cores, ten cores, and more (Sondhi and Ganesh n.d.) (Zeebaree et al. 2020). The multi-core processor is an integrated circuit (IC) with two or more processors linked to improve performance, reduce energy consumption, and handle multiple tasks more efficiently and simultaneously (Rouse 2013).

The multi-core processor can be classified into two types: homogeneous and heterogeneous. Symmetric multiprocessor (SMP) operating systems are usually implemented on monolithic multi-core processors to compute high-performance combinations. On the other hand, heterogeneous multi-core processors, which consist of various cores dedicated to specific applications, are better for embedded systems(Wei et al. 2011).

In recent years, CPU designers have moved from a multi-core architecture with multiple processing threads to a clock speed of multi-core processors (Rao, Prasad, and Venkateswarlu 2009). The core can be considered as one healer. The dual-core contains two internal processors, which are produced by a single chip. The quad-core model has four processors, two dual-processors, made on one chip. More cores are useful for a variety of tasks. Single-thread applications can use only one kernel, leaving any other procedure idle. Core i3 processors have four cores, and i5 and i7 has four cores (Rao, Moturi, and KLEF 2018). 


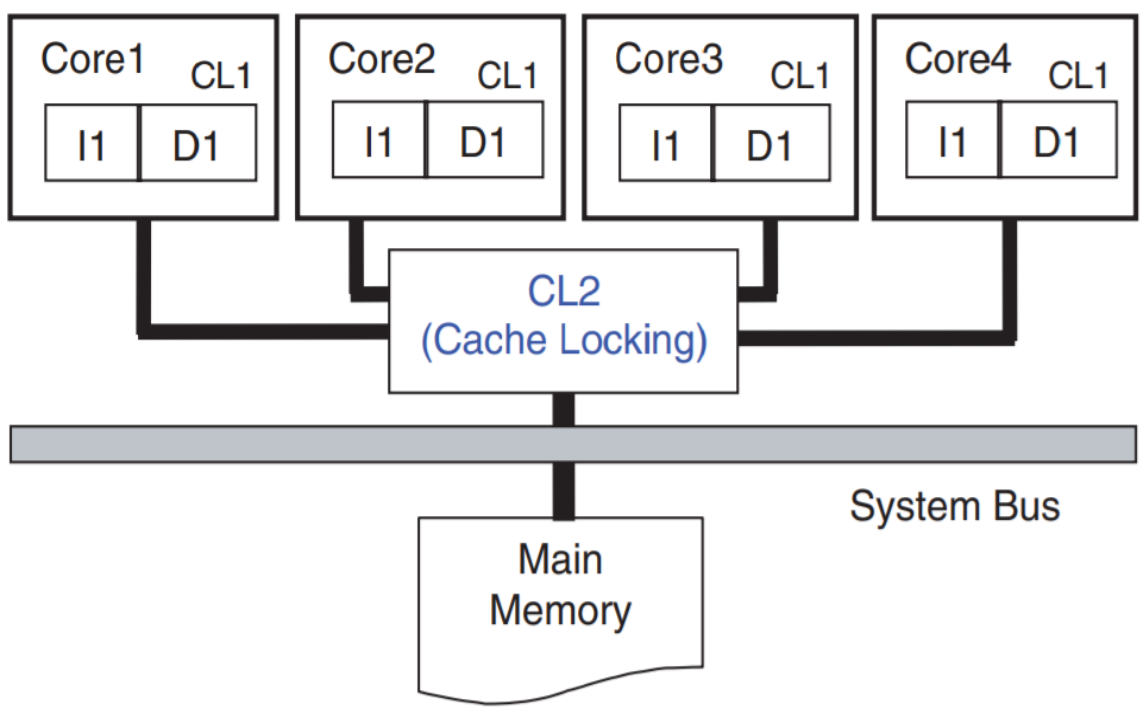

Figure (5): multi-core distributed system (Asaduzzaman and Gunasekara 2012).

\section{Literature Review}

V. Sklyarov, et. al. (Sklyarov et al. 2016) in 2016, explored distributed computing systems that can be used efficiently to process frequently requested information in electronic, environmental, medical and biological applications. Pre-processing can be done in very parallel accelerators that are set to reconfigurable devices. The core of the accelerator is a sort/search network that is implemented either in FPGA or in a programmable chip-based system (such as ZYNQ devices). Data is sent to the computer via the high-bandwidth $\mathrm{PCl}$-Express bus.

B. Sreevidya, et. al. (Sreevidya, Rajesh, and Mamatha 2018) in 2018, worked on determination of sensor network performance after on-demand accuracy using an RSA-based security scheme that allows fake data to enter the wireless sensor network(WSN) using NS2 emulation. The RSA algorithm applied in the modified scheme provides large system security than the MAC algorithm of the BECAN scheme. The MAC algorithm only denoted system authentication, but the RSA algorithm constructed the system more security with public key encryption technology. 
S. Phoemphon, et. al. (Phoemphon, So-In, and Nguyen 2018) in 2018, studied the possible integration of two computing technologies, fuzzy logic (FL) and extreme learning machines (ELMs), with the aim of improving the estimated localization accuracy, taking into account the above factors. Localization is one of the major challenges encountered in wireless sensor networks, especially in the non-attendance of such GPS installation equipment. Unlike ELMs, FL methods provide high accuracy under limited node density and coverage conditions.

M. Hossain, et. al. (Hossain et al. 2018) in 2018, addressed the designed network measured latency and throughput compared to the interactive real-time online (ROIA) multi-box schedule and NOX. The main technology of SDN application is data level and management level separation, and network virtualization through programming. The total amount of time a user can respond is called the response time. The transfer rate is the exchange rate at which the network transmits data.

A. Rauniyar, et. al. (Rauniyar, Engelstad, and Moen 2018) in 2018, proposed a new distributed localization algorithm is using SL-PSO based social learning for the Internet of Things. With the SLPSO algorithm, the method aims to precisely localize diffuse sensor nodes and reduce computational complexity that will further enhance the lifespan of these sensing nodes with limited resources. Extensive simulations are performed to demonstrate the effective performance of the SL-PSO algorithm in fine localization.

Y. Liu, et. al. (Liu et al. 2019) in 2019, proposed a lightweight block-chain system called a lightweight chain that is resource-efficient and suitable for energy Industrial Internet of Things (IloT) scenarios. In particular, a green agreement mechanism called symmetric multicore processer (SMP) to facilitate collaboration among $\underline{\text { lloT }}$ devices, a light data structure called light-block (LB) to identify broadcast content. Moreover, they designed a new block-chain to avoid unlimited overhead growth without affecting the block-chain backlog.

F. Al-Wesabi, et. al. (Al-Wesabi, Iskandar, and Ghilan 2019) in 2019, expanded the work of the E-Avala approximation to improving the overall performance of the workstudy by increasing power recovery capabilities. The work-study was executed and 
compared with the previous study using square modelling with different configuration parameters. The comparative results show that the proposed study has allowed for better improvement with recycling capabilities at different levels.

L. Tan, et. al. (Tan, Zhao, and Zhang 2019) in 2019, demonstrated using iTrace to collect effects on iOS. This creates opportunities for many types of searches that were previously impossible to perform on iOS. The iTrace Ultra Speed also allows for timesensitive analysis. The collected system calls for effects for commonly used applications and summarizes and displays their results from these effects. They hoped to highlight application behaviour and open up areas for further investigation.

F. Noor, et. al. (Noor, Ibrahim, and AlKhattab 2020) in 2020, proposed the parallel distributed bat algorithm (PDBA) using the Message Passing Interface (MPI) of the computer group in the programming $C$ language. PDBA time and complexity are determined. Also, the results were presented in terms of speed, efficiency, time completed number of times to perform the fitness function. An algorithm is an optimization algorithm that is workable and efficient to obtain the best approximate solutions to nonlinear problems.

M. Bhatia, et. al. (Bhatia, Sood, and Kaur 2020) in 2020, proposed a quantitative approach to the planning of homogeneous functions in fog-based applications. In particular, the scale of a given node is determined by calculating the node computational index to estimate the computational power of the fog computing nodes. Moreover, the $\mathrm{QCl}$ neural network model is proposed to optimize the node to handle the real-time mock task. Comparative analysis was performed using advanced scheduling models such as heterogeneous completion time, Min-Max, and Round Robin for comparative analysis to determine performance improvements.

K. Warasup, et. al. (Warasup, Hamamura, and Pattaramalai 2020) in 2020, introduced a new MAC protocol that enhances network performance for wireless LAN with Multiple

Packet Reception (MPR) capability. The main feature of their proposed protocol is that multiple asynchronous RTS transmissions are allowed. As a result, the method get a higher probability of packet transmission when the network is operating at the 
maximum MPR capacity. Moreover, they are developed a mathematical model to evaluate the proposed protocol.

D. K. Kotary, et. al. (Kotary and Nanda 2020) in 2020, compared with Diffusion Particle Swarm Optimization (DPSO), Diffusion Whale Optimization Agorithm (DWOA), Dffusion Elephant Herding Optimization (DEHO), Diffused K-Mans (DK-Mans) Dunn Index, and Outline Index In terms of time complexity. Performed on two real groups of artificial data sets. The minimum average Euclidean deviation (EAD) for improving farm butterfly flame for agricultural farm data proportion compared to DPSO, DWOA, DEHO, and DK-Mans is $7.16 \%, 3.25 \%, 5.24 \%$, and $21.70 \%$.

Bianco, et. al. (Bianco 2020) in 2020, discussed the design and development of FPGAbased NIC enabling us to overcome performance barriers bottlenecks and Lack of flexibility in commercial NICs. Network interface cards (NICs) are gaining more and more attention in the research community as they can provide several gigabytes of transmission speeds per packet, a performance similar to low-to-mid-range routers. The effectiveness of the proposed approach and limits was comprehensively discussed.

P. Neelima, et. al. (Neelima and Reddy 2020) in 2020, introduced the ADA-based, multi-load balancing method, and this system is being tested through simulation experiments. They have planned an approach of the design to deliver a well-balanced load on virtual machines deliver them on time at the lowest cost. Moreover, the proposed algorithm demonstrates the ADA's ability to improve task scheduling and resource allocation in the cloud computing environment. To assess the performance of the proposed system, three problematic cases are analysed.

S. Yousefi, et. al. (Yousefi et al. 2020) in 2020, introduced a new route planning mechanism for collecting data on the Internet. The proposed mechanism includes two basic states. Gather data on the Internet of Things using the idea of mobile software agents. The first step is to integrate loT devices. The Group leaders assembled groups by assigning mobile agents to a wedge-based operation. The basic purpose of the second period is to provide a path mapping to each mobile factor of group leaders to effectively collect data through the Markov Determination Process (MDP). 
F. Shahid, et. al. (Shahid, Khan, and Jeon 2020) in 2020, proposed Distributed Ledger for Internet of Things (DL-for-loT) addresses the challenge of integrating the two technologies: distributed book and Internet of things. Notable features of DL-for-loT include transaction hierarchy, floor cuts, lightweight compatibility, and quantitatively secure digital signatures. The basic building block of DL-for-loT is a new one-time signature (OTS) method called DL-OTS. By comparing DL-OTS with general one-time signature schemes, they concluded that DL-OTS is a compact and high-speed energysaving signature scheme.

X. Zhao, et. al. (Zhao et al. 2020) in 2020, designed and developed based on distributed heterogeneous medical data a system to share and merge the distributed medical data and apply it to some internal hospitals. Therefore, a series of smart medical information network platform system is created so that patients can enjoy the service. High quality, safe, and proper diagnosis and dealing are based on little waiting times and vital medical costs. Finally, the results of questionnaires and interviews concluded that they were compared to previous treatments.

S. Dhingra, et. al. (Dhingra et al. 2020) in 2020, introduced a cloud fog for real-time analysis of the network of basic cloud services for traffic monitoring base to overcome the clogging latency limits. Therefore, proposed to implement a prototype Smart Traffic Monitoring System (STMS) and it is designed for signal traffic monitoring of overcrowding. It can also be adapted to detect road accidents that require immediate assistance in case of congestion. Within this framework, the small computer on the module acts as a fog node, collecting real-time data from geographically dispersed sensors, moving it to the cloud for storage and processing.

Parra, et. al. (Parra et al. 2020) in 2020, proposed cloud distributed deep learning framework for detecting the phishing attacks. The model consists of two main security mechanisms that operate cooperatively: first, a distributed convolutional neural network (DCNN) model included as an add-on partial security for the Internet of Things device to detect phishing and denial of distributed service application (DDoS). Second, a cloud-based Long-Short Term Memory (LSTM) model hosted on the backside to detect Botnet attacks and CNN embedding to detect phishing attacks 
distributed across many loT devices. Distributed CNN model, included in the ML engine of customer's loT device.

Y. Gao, et. al. (Gao et al. 2020) in 2020, evaluated and compared FL and SplitNN IoT settings in the real world in terms of learning performance and overall hardware application. considered a wide variety of data sets, different model structures, multiple clients, and different performance metrics. For learning performance, determined by model accuracy and convergence velocity measures, evaluated the empirically for both FL and SplitNN under different types of data distributions such as unbalanced, non-independent, and identically distributed data (other than IID)

Y. Ren, et. al. (Ren et al. 2020) in 2020, introduced the innovative DCOMB (Dual Combination Bloom Filter) method to convert the processing power of bitcoin mining into query processing power. In addition, the use of the DCOMB method to create a block chain based loT data query model. This model combines the flow of loT data with a block chain of the timing chain, improving the interoperability and versatility of the data of the loT database system.

W. Yánez, et. al. (Yánez et al. 2020) in 2020, proposed a new context-sensitive mechanism for distributing chain data across loT blockchain systems. In particular, they developed the data controller based on tasteless logic to calculate the cost allocation value for each data request, taking into account multiple context parameter data network quality and their chain distribution. Moreover, introduced how the design and perception of improves the architectural styles of the two common uses of the Internet of Things (block, fog).

\section{Discussion}

This part depends on the details explained in table 1. All the above literature used different techniques in distributed systems and the significant result. For example. The first literature V. Sklyarov, et al. the Scheduling algorithms used and the results of pre-processing, statistical treatment, analysis of existing, acquired groups and data mining. Also, B. Sreevidya, et al. the MAC and RSA algorithm used and the result indicate that schema performance is better than the current schemes that provide 


\section{QALAAI ZANISTSCIENTIFIC JOURNAL}

A Scientific Quarterly Refereed Journal Issued by Lebanese French University - Erbil, Kurdistan, Iraq

Vol. (6), No (4), Autumn 2021

ISSN 2518-6566 (Online) - ISSN 2518-6558 (Print)

either data integrity or sender authentication. Furthermore, S. Phoemphon, et al. the ELM algorithm used and the result derivatives and improvements, Fuzzy Centroid and ELMs.

In addition, each piece of literature uses a different operating system and different versions. For example L. Tan, et al. used iOS 4.1 and Android 2.3.6, G. D. L. T. Parra used Linux (Ubuntu 16.04) and Y. Ren, et al. used windows 10. On the other hand, the programming language is the crucial part of any paper, and all the above literature uses different programming languages, such as Matlab, Java, Python, $\mathrm{C}++$. Also, the network is the tower part for the distributed system because the literature shows that if they use a good network and fast internet, they will get a good outcome. Finally, all the above literature used an excellent technique and found a significant result: one is the best and gets high performance for system displayed at the last part of this paper. 


\section{QALAAI ZANISTSCIENTIFIC JOURNAL}

A Scientific Quarterly Refereed Journal Issued by Lebanese French University - Erbil, Kurdistan, Iraq

Vol. (6), No (4), Autumn 2021

ISSN 2518-6566 (Online) - ISSN 2518-6558 (Print)

Table (1): Analysis of surveyed researches

\begin{tabular}{|c|c|c|c|c|c|c|}
\hline Literature & Technique & Significant Results & $\begin{array}{l}\text { Operating } \\
\text { System }\end{array}$ & CPU type & Programing & $\begin{array}{l}\text { Networking } \\
\text { approach }\end{array}$ \\
\hline $\begin{array}{l}\text { (Sklyarov et } \\
\text { al. 2016) }\end{array}$ & $\begin{array}{l}\text { Scheduling } \\
\text { algorithms }\end{array}$ & $\begin{array}{l}\text { The results of pre-processing, statistical treatment, } \\
\text { analysis of existing, acquired groups and data } \\
\text { mining. }\end{array}$ & 32-bit Linux & dual-core & C language & Wired \\
\hline $\begin{array}{l}\text { (Sreevidya et } \\
\text { al. 2018) }\end{array}$ & $\begin{array}{l}\text { MAC and RSA } \\
\text { algorithm }\end{array}$ & $\begin{array}{l}\text { Results indicate that the schema performance is } \\
\text { better than the current schemes that provide either } \\
\text { data integrity or sender authentication. }\end{array}$ & Linux & - & $\begin{array}{c}\mathrm{C}++ \\
\text { language }\end{array}$ & $\begin{array}{l}\text { Wired and } \\
\text { wireless }\end{array}$ \\
\hline $\begin{array}{l}\text { (Phoemphon } \\
\text { et al. 2018) }\end{array}$ & ELM algorithm & $\begin{array}{l}\text { Traditional free domain localization plans consisting } \\
\text { of Centroid, derivatives and improvements, Fuzzy } \\
\text { Centroid and ELMs. }\end{array}$ & Windows & $\begin{array}{l}\text { Quad Core } \\
\qquad \text { (TM) } \\
\text { Q8400 }\end{array}$ & $\begin{array}{l}\text { MATLAB } \\
\text { language }\end{array}$ & Wireless \\
\hline $\begin{array}{l}\text { (Hossain et } \\
\text { al. 2018) }\end{array}$ & $\begin{array}{l}\text { SDN and QOS } \\
\text { technique }\end{array}$ & $\begin{array}{l}\text { Easily configure traffic without touching any } \\
\text { individual keys and services needed in the network. }\end{array}$ & Linux & - & $\begin{array}{l}\text { PYTHON } \\
\text { language }\end{array}$ & Wired \\
\hline $\begin{array}{l}\text { (Rauniyar et } \\
\text { al. 2018) }\end{array}$ & $\begin{array}{l}\text { SL-PSO } \\
\text { algorithm }\end{array}$ & $\begin{array}{c}\text { Significantly reduced mean localization error } \\
\text { compared to the optimization of conventional } \\
\text { particle swarm (PSO). }\end{array}$ & $\begin{array}{c}\text { Windows } 7 \\
\text { 64-bit }\end{array}$ & $\begin{array}{l}\text { Intel Core } \\
\text { i7-6500 }\end{array}$ & $\begin{array}{l}\text { MATLAB } \\
\text { R2016b }\end{array}$ & Wireless \\
\hline
\end{tabular}




\section{QALAAI ZANISTSCIENTIFIC JOURNAL}

A Scientific Quarterly Refereed Journal Issued by Lebanese French University - Erbil, Kurdistan, Iraq

Vol. (6), No (4), Autumn 2021

LF U

ISSN 2518-6566 (Online) - ISSN 2518-6558 (Print)

\begin{tabular}{|c|c|c|c|c|c|c|}
\hline $\begin{array}{l}\text { (Liu et al. } \\
\text { 2019) }\end{array}$ & $\begin{array}{l}\text { Light Block and } \\
\text { UBOF algorithm }\end{array}$ & $\begin{array}{l}\text { The individual computational cost can reduce to } \\
39.32 \% \text { and speed up the block generation by up to } \\
74.06 \% \text {. }\end{array}$ & $\begin{array}{l}\text { GUN/Linux } \\
\text { 4.8.0-36 }\end{array}$ & $\begin{array}{l}\text { Intel Xeon } \\
2.5\end{array}$ & Python & Wireless \\
\hline $\begin{array}{l}\text { (Al-Wesabi et } \\
\text { al. 2019) }\end{array}$ & $\begin{array}{l}\text { E-Avala } \\
\text { algorithm }\end{array}$ & $\begin{array}{l}\text { It gave a better performance with different levels of } \\
\text { processing capabilities. }\end{array}$ & Windows 7 & $\begin{array}{c}\text { Intel } \\
\text { Core }^{\mathrm{TM}} \mathrm{i5} \\
\mathrm{M} 450 / 2.40\end{array}$ & $\begin{array}{l}\text { JAVA } \\
\text { language }\end{array}$ & - \\
\hline $\begin{array}{l}\text { (Tan et al. } \\
\text { 2019) }\end{array}$ & iTrace & $\begin{array}{c}\text { The difference in the frameworks used or } \\
\text { developed using a different (logical) application to } \\
\text { achieve the same function. }\end{array}$ & $\begin{array}{l}\text { iOS } 4.1 \text { and } \\
\text { Android 2.3.6 }\end{array}$ & $\begin{array}{l}\text { Cortex A8, } \\
1 \mathrm{GHz}\end{array}$ & Clanguage & $\begin{array}{l}\text { Mobile(wire } \\
\text { less) }\end{array}$ \\
\hline $\begin{array}{l}\text { (Noor et al. } \\
\text { 2020) }\end{array}$ & Bat algorithm & $\begin{array}{l}\text { Effective for optimization problems with large } \\
\text { population size and extensive search space }\end{array}$ & Linux & - & C language & Wireless \\
\hline $\begin{array}{l}\text { (Bhatia et al. } \\
\text { 2020) }\end{array}$ & $\begin{array}{l}\text { QCl-load } \\
\text { scheduling } \\
\text { algorithm }\end{array}$ & $\begin{array}{l}\text { The presented approach is extremely effective in } \\
\text { optimizing the contract computing of fog in } \\
\text { wireless applications in real-time }\end{array}$ & - & $\begin{array}{l}\text { Intel Core } \\
\text { TM i7- } \\
\text { 4710MQ }\end{array}$ & $\begin{array}{c}\text { JAVA } \\
\text { language }\end{array}$ & Wireless \\
\hline $\begin{array}{l}\text { (Warasup et } \\
\text { al. 2020) }\end{array}$ & MU-MIMO & $\begin{array}{l}\text { High bandwidth transmission potential when } \\
\text { network is running at full MPR capacity }\end{array}$ & Windows & - & $\begin{array}{l}\text { MATLAB } \\
\text { language }\end{array}$ & Wireless \\
\hline $\begin{array}{l}\text { (Kotary and } \\
\text { Nanda 2020) }\end{array}$ & $\begin{array}{c}\text { DMFO } \\
\text { algorithm }\end{array}$ & Increasing sensor data size or sensor node number. & $\begin{array}{c}\text { Windows } 8 \\
\text { (64) bit }\end{array}$ & Core i3 & $\begin{array}{l}\text { MATLAB } \\
\text { language }\end{array}$ & Wireless \\
\hline
\end{tabular}




\section{QALAAI ZANISTSCIENTIFIC JOURNAL}

A Scientific Quarterly Refereed Journal Issued by Lebanese French University - Erbil, Kurdistan, Iraq

Vol. (6), No (4), Autumn 2021

LF U

ISSN 2518-6566 (Online) - ISSN 2518-6558 (Print)

\begin{tabular}{|c|c|c|c|c|c|c|}
\hline (Bianco 2020) & $\begin{array}{l}\text { scheduling } \\
\text { algorithm }\end{array}$ & $\begin{array}{c}\text { Processed and managed in the software by the } \\
\text { operating system, thereby reducing charging } \\
\text { performance. }\end{array}$ & Linux & $\mathrm{PCl}-\mathrm{X}$ core & $\begin{array}{l}\text { LOGIC } \\
\text { design }\end{array}$ & $\begin{array}{c}\text { Wireless } \\
\text { packet } \\
\text { network } \\
\text { (WPN) }\end{array}$ \\
\hline $\begin{array}{l}\text { (Neelima and } \\
\text { Reddy 2020) }\end{array}$ & $\begin{array}{c}\text { Adaptive } \\
\text { Dragonfly } \\
\text { algorithm (ADA) }\end{array}$ & $\begin{array}{l}\text { A better approach achieves the result of load } \\
\text { balancing compared to other approaches. }\end{array}$ & $\begin{array}{c}\text { Windows } 7 \\
\text { (64)bit }\end{array}$ & dual-core & Java & - \\
\hline $\begin{array}{l}\text { (Yousefi et al. } \\
\text { 2020) }\end{array}$ & $\begin{array}{l}\text { k-medoids } \\
\text { algorithm }\end{array}$ & $\begin{array}{c}\text { The proposed mechanism improves power } \\
\text { consumption, data transmission delay, and loT } \\
\text { reliability }\end{array}$ & Windows 7 & $\begin{array}{l}\text { Intel }(\mathrm{R}) \\
\text { Core i7- } \\
3520 \mathrm{M}\end{array}$ & - & Wireless \\
\hline $\begin{array}{l}\text { (Shahid et al. } \\
\text { 2020) }\end{array}$ & $\begin{array}{l}\text { DL-OTS } \\
\text { algorithm }\end{array}$ & $\begin{array}{c}76 \% \text { reduction at the time of signature and } 48.7 \% \\
\text { energy savings compared to Winter-Nitz-OTS } \\
\text { scheme. }\end{array}$ & $\begin{array}{l}\text { Windows } 8.1 \\
\text { 32-bit }\end{array}$ & $\begin{array}{l}\text { Intel Core } \\
\text { i5 }\end{array}$ & Python & Wireless \\
\hline $\begin{array}{l}\text { (Zhao et al. } \\
\text { 2020) }\end{array}$ & AES algorithm & $\begin{array}{l}\text { The participation of smart medicine among the } \\
\text { general public increased by about } 79 \%\end{array}$ & $\begin{array}{l}\text { Linux } \\
\text { (Ubuntu } \\
\text { 12.04) } 32 \text { bit }\end{array}$ & $\begin{array}{c}\text { Intel } \\
\text { Q9300 }\end{array}$ & Python & Wireless \\
\hline $\begin{array}{l}\text { (Dhingra et } \\
\text { al. 2020) }\end{array}$ & $\begin{array}{c}\text { STMS } \\
\text { technique }\end{array}$ & $\begin{array}{l}\text { Improved cloud platform performance in terms of } \\
\text { reduced response time and increased bandwidth. }\end{array}$ & $\begin{array}{l}\text { Windows } \\
\text { (64) bit }\end{array}$ & dual-core & $\begin{array}{l}\text { (Arduino } \\
\text { IDE) Java }\end{array}$ & Wireless \\
\hline
\end{tabular}




\section{QALAAI ZANISTSCIENTIFIC JOURNAL}

A Scientific Quarterly Refereed Journal Issued by Lebanese French University - Erbil, Kurdistan, Iraq

Vol. (6), No (4), Autumn 2021

LF U

ISSN 2518-6566 (Online) - ISSN 2518-6558 (Print)

\begin{tabular}{|c|c|c|c|c|c|c|}
\hline $\begin{array}{c}\text { (Parra et al. } \\
\text { 2020) }\end{array}$ & $\begin{array}{l}\text { DCNN and } \\
\text { LSTM model }\end{array}$ & $\begin{array}{l}\text { Detection results for specific attacks when the } \\
\text { model is trained with benign and malicious data }\end{array}$ & $\begin{array}{l}\text { Linux } \\
\text { (Ubuntu } \\
16.04 \text { ) }\end{array}$ & $\begin{array}{l}\text { Intel Xeon } \\
\text { e5-2670 } \\
\text { V3 }\end{array}$ & Python & Wireless \\
\hline $\begin{array}{l}\text { (Gao et al. } \\
\text { 2020) }\end{array}$ & $\begin{array}{l}\text { FL and Split-NN } \\
\text { model }\end{array}$ & $\begin{array}{c}\text { Our experimental results of learning performance } \\
\text { at Split-NN research questions for future } \\
\text { investigations }\end{array}$ & windows 10 & $\begin{array}{l}\text { Intel core } \\
\text { i7- } \\
7700 \mathrm{HQ}\end{array}$ & Python & Wireless \\
\hline $\begin{array}{l}\text { (Ren et al. } \\
\text { 2020) }\end{array}$ & $\begin{array}{l}\text { DCOMB } \\
\text { model }\end{array}$ & $\begin{array}{l}\text { The results showed that the random read } \\
\text { performance of } D C O M B \text { query is higher than } C O M B\end{array}$ & windows 10 & Intel i3 & Java & - \\
\hline $\begin{array}{c}\text { (Yánez et al. } \\
\text { 2020) }\end{array}$ & loT-blockchain & $\begin{array}{l}\text { Experimental results indicate that realizing a data } \\
\text { allocation mechanism improves network latency, } \\
\text { blockchain storage and reduces power } \\
\text { consumption. }\end{array}$ & Windows 7 & $\begin{array}{l}\text { Intel Core } \\
2 \text { Duo }\end{array}$ & $\begin{array}{l}\text { Java and } \\
\text { MySQL }\end{array}$ & Wireless \\
\hline
\end{tabular}




\section{QALAAI ZANISTSCIENTIFIC JOURNAL \\ A Scientific Quarterly Refereed Journal Issued by Lebanese French University - Erbil, Kurdistan, Iraq \\ Vol. (6), No (4), Autumn 2021 \\ ISSN 2518-6566 (Online) - ISSN 2518-6558 (Print)}

\section{Conclusion}

Distributed systems are designed separately from the core network, making the worst assumptions. The multicore processor can be classified into two types: homogeneous and heterogeneous. This paper reviewed of Performance Impact of DistributedMemory Parallel Processing Approach on Performance Enhancing of MulticomputerMulticore Systems. Also, number of methods been introduced which used Impact of Distributed-Memory Parallel Processing Approach on Performance Enhancing of Multicomputer-Multicore Systems. Adding to that, the best methods been explained, with focusing on speed for performance enhancing of multicore in the distributed systems.

Depending on the details explained in Table I in the discussion section, it can be concluded that the best methods were those which depended by Y. Liu et al. and F. Shahid, et al. Firstly, Liu et al. used an operating system named gun/Linux 4.8.0-36, intel Xeon 2.5, python programming language. The outcome of the proposed method made a time reduction by $39.32 \%$ and speeded up the block generation up to $74.06 \%$ of individual computational cost. Secondly, F. Shahid et al. used an operating system named Windows 8.1 32-bit Intel core i5. The programming language is python, and result, it can reduce $76 \%$ at the time of signature and $48.7 \%$ energy savings compared to winternitz-ots scheme.

\section{References:}

Agarwal, V. 2004. "Fault Tolerance in Distributed Systems, I. Institute of Technology Kanpur."

Al-Wesabi, Fahd, Huda Iskandar, and Mokhtar Ghilan. 2019. "Improving Performance in Component Based Distributed Systems." EAI Endorsed Transactions on Scalable Information Systems 6(22).

Amir, Yair, Yongdae Kim, Cristina Nita-Rotaru, John L. Schultz, Jonathan Stanton, and Gene Tsudik. 2004. "Secure Group Communication Using Robust Contributory Key Agreement." IEEE Transactions on Parallel and Distributed Systems 15(5):468-80.

Anton, B., and A. Norbert. 2016. "Peer to Peer System Deployment." Acta Electrotech. Inform 16:11-14. 


\section{QALAAI ZANISTSCIENTIFIC JOURNAL \\ A Scientific Quarterly Refereed Journal Issued by Lebanese French University - Erbil, Kurdistan, Iraq \\ Vol. (6), No (4), Autumn 2021 \\ ISSN 2518-6566 (Online) - ISSN 2518-6558 (Print)}

Asaduzzaman, Abu, and Govipalagodage H. Gunasekara. 2012. "A Way Cache Locking Scheme

Supported by Knowledge Based Smart Preload Effective for Low-Power Multicore Electronics." Journal of Low Power Electronics 8(5):552-64.

Bansal, Sanjay, Sanjeev Sharma, and Ishita Trivedi. 2011. "A Detailed Review of FaultTolerance Techniques in Distributed System." International Journal on Internet \& Distributed Computing Systems 1(1).

Bhatia, Munish, Sandeep K. Sood, and Simranpreet Kaur. 2020. "Quantumized Approach of Load Scheduling in Fog Computing Environment for loT Applications." Computing 1-19.

Bianco, Michele Petracca Robert Birke Andrea. 2020. "HERO: High-Speed Enhanced Routing

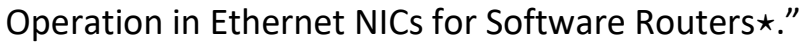

Blake, Geoffrey, Ronald G. Dreslinski, and Trevor Mudge. 2009. "A Survey of Multicore Processors." IEEE Signal Processing Magazine 26(6):26-37.

Bridges, Matthew J., Neil Vachharajani, Yun Zhang, Thomas Jablin, and David I. August. 2008. "Revisiting the Sequential Programming Model for the Multicore Era." IEEE Micro 28(1):12-20.

Chen, Xin, Xubin He, He Guo, and Yuxin Wang. 2011. "Design and Evaluation of an Online Anomaly Detector for Distributed Storage Systems." J. Softw. 6(12):2379-90.

Dhingra, Swati, Rajasekhara Babu Madda, Rizwan Patan, Pengcheng Jiao, Kaveh Barri, and Amir H. Alavi. 2020. "Internet of Things-Based Fog and Cloud Computing Technology for Smart Traffic Monitoring." Internet of Things 100175.

Dino, Hivi Ismat, Subhi R. M. Zeebaree, Omar M. Ahmad, Hanan M. Shukur, Rizgar R. Zebari, and Lailan M. Haji. n.d. "Impact of Load Sharing on Performance of Distributed Systems Computations."

Fiat, Amos, and Jared Saia. 2002. "Censorship Resistant Peer-to-Peer Content Addressable Networks." Pp. 94-103 in SODA. Vol. 2.

Gao, Yansong, Minki Kim, Sharif Abuadbba, Yeonjae Kim, Chandra Thapa, Kyuyeon Kim, Seyit A. Camtepe, Hyoungshick Kim, and Surya Nepal. 2020. "End-to-End Evaluation of Federated Learning and Split Learning for Internet of Things." ArXiv Preprint ArXiv:2003.13376.

Grudenić, Igor, and Nikola Bogunović. 2009. "Computer Cluster and Grid Simulator." in MIPRO 1134 


\section{QALAAI ZANISTSCIENTIFIC JOURNAL \\ A Scientific Quarterly Refereed Journal Issued by Lebanese French University - Erbil, Kurdistan, Iraq \\ Vol. (6), No (4), Autumn 2021 \\ ISSN 2518-6566 (Online) - ISSN 2518-6558 (Print)}

2009.

Haji, Lailan M., Subhi R. M. Zeebaree, Omar M. Ahmed, Amira B. Sallow, Karwan Jacksi, and Rizgar R. Zeabri. 2020. "Dynamic Resource Allocation for Distributed Systems and Cloud Computing."

Harinath, Depavath, K. Ramesh Babu, P. Satyanarayana, and M. V. Ramana Murthy. 2015. "Enhancing Security Using Video Steganography and WaterMarking." European Journal of Applied Sciences 3(5):1.

Hossain, Md, Mohammad Nowsin Amin Sheikh, Shawon Rahman, Sujan Biswas, and Md Ariful Islam Arman. 2018. "Enhancing and Measuring the Performance in Software Defined Networking." International Journal of Computer Networks \& Communications (IJCNC) 10(5).

Kaur, Ramanjyot, Tajinder Kaur, and Harpreet Kaur. 2013. "Scheduling in Grid Computing Environment." International Journal of Advanced Research in Computer Science and Software Engineering 3(6):455-58.

Kotary, Dinesh Kumar, and Satyasai Jagannath Nanda. 2020. "Distributed Robust Data Clustering in Wireless Sensor Networks Using Diffusion Moth Flame Optimization." Engineering Applications of Artificial Intelligence 87:103342.

Lakshmanan, Subramanian, Mustaque Ahamad, and H. Venkateswaran. 2003. "Responsive Security for Stored Data." IEEE Transactions on Parallel and Distributed Systems 14(9):818-28.

Liu, Yinqiu, Kun Wang, Yun Lin, and Wenyao Xu. 2019. "\$|mathsf \{LightChain $\}$ \$: A Lightweight Blockchain System for Industrial Internet of Things." IEEE Transactions on Industrial Informatics 15(6):3571-81.

Lu, Gang, Jianfeng Zhan, Xinlong Lin, Chongkang Tan, and Lei Wang. 2016. “On Horizontal Decomposition of the Operating System." ArXiv Preprint ArXiv:1604.01378.

McCool, Michael D. 2008. "Scalable Programming Models for Massively Multicore Processors." Proceedings of the IEEE 96(5):816-31.

Neelima, P., and A. Rama Mohan Reddy. 2020. "An Efficient Load Balancing System Using Adaptive Dragonfly Algorithm in Cloud Computing." Cluster Computing 1-9.

Noor, Fazal, Abdulghani Ibrahim, and Mohammed M. AlKhattab. 2020. “Performance of 1135 
Parallel Distributed Bat Algorithm Using MPI on a PC Cluster." Annals of Emerging Technologies in Computing (AETiC) 4(1).

Parra, Gonzalo De La Torre, Paul Rad, Kim-Kwang Raymond Choo, and Nicole Beebe. 2020. "Detecting Internet of Things Attacks Using Distributed Deep Learning." Journal of Network and Computer Applications 102662.

Phoemphon, Songyut, Chakchai So-In, and Tri Gia Nguyen. 2018. "An Enhanced Wireless Sensor Network Localization Scheme for Radio Irregularity Models Using Hybrid Fuzzy Deep Extreme Learning Machines." Wireless Networks 24(3):799-819.

Ports, Dan R. K., Jialin Li, Vincent Liu, Naveen Kr Sharma, and Arvind Krishnamurthy. 2015. "Designing Distributed Systems Using Approximate Synchrony in Data Center Networks." Pp. 43-57 in 12th \{USENIX\} Symposium on Networked Systems Design and Implementation (\{NSDI\} 15).

Puttaswamy, Krishna P. N., Heather Zheng, and Ben Y. Zhao. 2008. "Securing Structured Overlays against Identity Attacks." IEEE Transactions on Parallel and Distributed Systems 20(10):1487-98.

Ramanathan, R. M. 2006. "Intel Multi-Core Processors: Making the Move to Quad-Core and Beyond." Technology@ Intel Magazine 4(9):2-4.

Rao, S. N. Tirumala, Sireesha Moturi, and Vaddeswaram KLEF. 2018. "SCALABILITY OF MEMORY MAPPED K-MEANS ALGORITHM WITH HYPER-THREADED PROCESSORS." International Journal of Pure and Applied Mathematics 120(6):4179-92.

Rao, S. N. Tirumala, E. V Prasad, and N. B. Venkateswarlu. 2009. "A Scalable K-Means Clustering Algorithm on Multi-Core Architecture." Pp. 1-9 in 2009 Proceeding of International Conference on Methods and Models in Computer Science (ICM2CS). IEEE.

Rashid, Zryan Najat, Subhi R. M. Zebari, Karzan Hussein Sharif, and Karwan Jacksi. 2018. "Distributed Cloud Computing and Distributed Parallel Computing: A Review." Pp. 16772 in 2018 International Conference on Advanced Science and Engineering (ICOASE). IEEE.

Ratnasamy, Sylvia, Paul Francis, Mark Handley, Richard Karp, and Scott Shenker. 2001. "A Scalable Content-Addressable Network." Pp. 161-72 in Proceedings of the 2001 conference on Applications, technologies, architectures, and protocols for computer communications. 


\section{QALAAI ZANISTSCIENTIFIC JOURNAL \\ A Scientific Quarterly Refereed Journal Issued by Lebanese French University - Erbil, Kurdistan, Iraq \\ Vol. (6), No (4), Autumn 2021 \\ ISSN 2518-6566 (Online) - ISSN 2518-6558 (Print)}

Rauniyar, Ashish, Paal Engelstad, and Jonas Moen. 2018. "A New Distributed Localization Algorithm Using Social Learning Based Particle Swarm Optimization for Internet of Things." Pp. 1-7 in 2018 IEEE 87th Vehicular Technology Conference (VTC Spring). IEEE.

Ren, Yongjun, Fujian Zhu, Pradip Kumar Sharma, Tian Wang, Jin Wang, Osama Alfarraj, and Amr Tolba. 2020. "Data Query Mechanism Based on Hash Computing Power of Blockchain in Internet of Things." Sensors 20(1):207.

Rouse, Margaret. 2013. "Definition: Multi-Core Processor." TechTarget. Retrieved March 6.

Rowstron, Antony, and Peter Druschel. 2001. "Pastry: Scalable, Decentralized Object Location, and Routing for Large-Scale Peer-to-Peer Systems." Pp. 329-50 in IFIP/ACM International Conference on Distributed Systems Platforms and Open Distributed Processing. Springer.

Saafan, Ahmed. 2009. "Distributed Denial of Service Attacks: Explanation, Classification and Suggested Solutions." 23 March 2009)[Online]. Available: Http://Www. Exploitdb. Com.

Shahid, Furqan, Abid Khan, and Gwanggil Jeon. 2020. "Post-Quantum Distributed Ledger for Internet of Things." Computers \& Electrical Engineering 83:106581.

Shukur, Hanan, Subhi Zeebaree, Rizgar Zebari, Omar Ahmed, Lailan Haji, and Dildar Abdulqader. 2020. "Cache Coherence Protocols in Distributed Systems." Journal of Applied Science and Technology Trends 1(3):92-97.

Sklyarov, V., Artjom Rjabov, louliia Skliarova, and Alexander Sudnitson. 2016. "HighPerformance Information Processing in Distributed Computing Systems." International Journal of Innovative Computing, Information and Control 12(1):139-60.

Sondhi, Garima, and Abhinaya Ganesh. n.d. "An Overview of Multi Core Processors."

Sreevidya, B., M. Rajesh, and T. M. Mamatha. 2018. "Design and Development of an Enhanced Security Scheme Using RSA for Preventing False Data Injection in Wireless Sensor Networks." Pp. 225-36 in Ambient Communications and Computer Systems. Springer.

Van Steen, Maarten, and Andrew S. Tanenbaum. 2017. Distributed Systems. Maarten van Steen Leiden, The Netherlands.

Stoica, Ion, Robert Morris, David Karger, M. Frans Kaashoek, and Hari Balakrishnan. 2001. "Chord: A Scalable Peer-to-Peer Lookup Service for Internet Applications." ACM 


\section{QALAAI ZANISTSCIENTIFIC JOURNAL \\ A Scientific Quarterly Refereed Journal Issued by Lebanese French University - Erbil, Kurdistan, Iraq \\ Vol. (6), No (4), Autumn 2021 \\ ISSN 2518-6566 (Online) - ISSN 2518-6558 (Print)}

SIGCOMM Computer Communication Review 31(4):149-60.

Sweilam, N. H., H. M. Moharram, and Sameh Ahmed. 2012. "On the Parallel Iterative Finite Difference Algorithm for 2-D Poisson's Equation with MPI Cluster." Pp. MM-78 in 2012 8th International Conference on Informatics and Systems (INFOS). IEEE.

Tan, Lucas, Fuyao Zhao, and Xu Zhang. 2019. "15712 Advanced Operating and Distributed System Android and IOS Platform Study Final Report."

Wang, Chonggang, and Bo Li. 2003. "Peer-to-Peer Overlay Networks: A Survey." Department of Computer Science, The Hong Kong University of Science and Technology, Hong Kong 9.

Wang, Lizhe, Jie Tao, Gregor von Laszewski, and Holger Marten. 2010. "Multicores in Cloud Computing: Research Challenges for Applications." JCP 5(6):958-64.

Warasup, Kittipong, Masanori Hamamura, and Suwat Pattaramalai. 2020. "Performance Analysis for Asynchronous Requests in Wireless Networks with Multiple Packet Reception Capability." Wireless Personal Communications 1-23.

Wei, Ting-Ying, Zhi-liang Qiu, Chung-ping Young, and Da-Wei Chang. 2011. "Development of Heterogeneous Multi-Core Embedded Platform for Automotive Applications." in 2011 International Conference on Circuits, System and Simulation (IPCSIT). Vol. 7.

Yánez, Wendy, Redowan Mahmud, Rami Bahsoon, Yuqun Zhang, and Rajkumar Buyya. 2020. "Data Allocation Mechanism for Internet-of-Things Systems With Blockchain." IEEE Internet of Things Journal 7(4):3509-22.

Yousefi, Shamim, Farnaz Derakhshan, Hadis Karimipour, and Hadi S. Aghdasi. 2020. "An Efficient Route Planning Model for Mobile Agents on the Internet of Things Using Markov Decision Process." Ad Hoc Networks 98:102053.

Zeebaree, Subhi R. M., Lailan M. Haji, Imad Rashid, Rizgar R. Zebari, Omar M. Ahmed, Karwan Jacksi, and Hanan M. Shukur. 2020. "Multicomputer Multicore System Influence on Maximum Multi-Processes Execution Time."

Zhao, Ben Yanbin, John Kubiatowicz, and Anthony D. Joseph. 2001. "Tapestry: An Infrastructure for Fault-Tolerant Wide-Area Location and Routing."

Zhao, Xin, Wei Xiao, Lu Wu, Zhigang Zhao, Jidong Huo, Shi Wang, Zhenhua Guo, and Dianmin Sun. 2020. "Intelligent City Intelligent Medical Sharing Technology Based on Internet of 


\section{QALAAI ZANISTSCIENTIFIC JOURNAL}

A Scientific Quarterly Refereed Journal Issued by Lebanese French University - Erbil, Kurdistan, Iraq

Vol. (6), No (4), Autumn 2021

ISSN 2518-6566 (Online) - ISSN 2518-6558 (Print)

Things Technology." Future Generation Computer Systems.

كاريكهرى جارهسهركردنى بهلاوكردنهوهى ميمورى بوّ باشتركردنى بهريّوهجونى

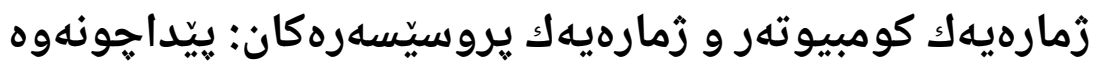

كورتكراوه: بهلاوكردنهوهى ميمورى بهكارديّت بو زانستهكان كومبيوتهر لهسهر شيّوازى سيستهى

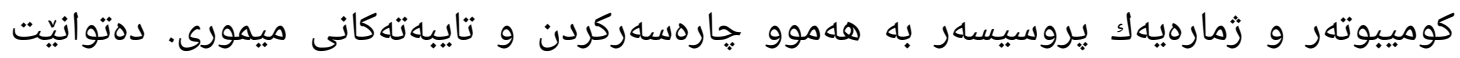

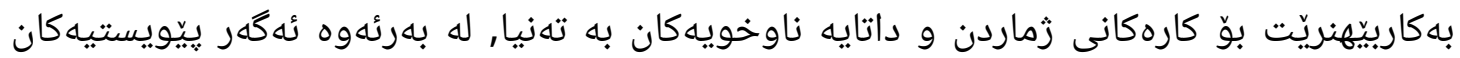

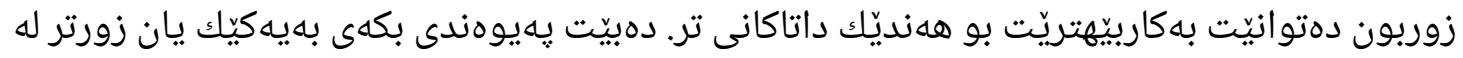

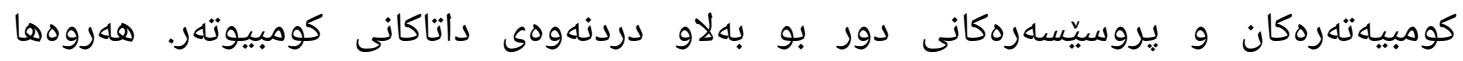

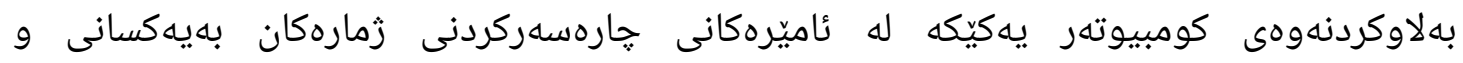

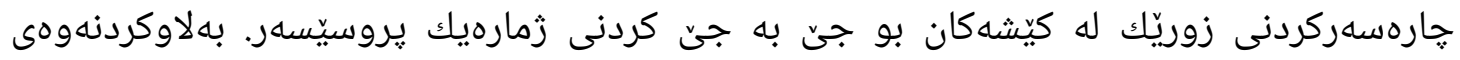

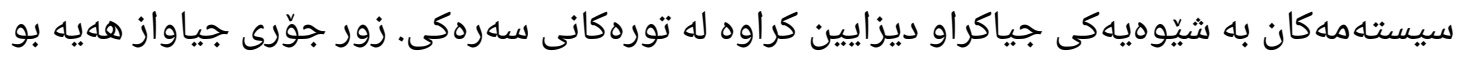

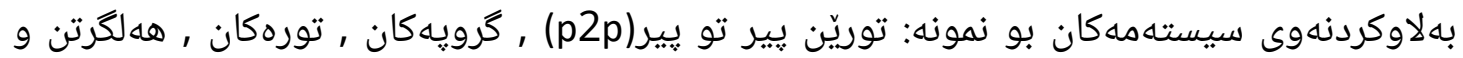

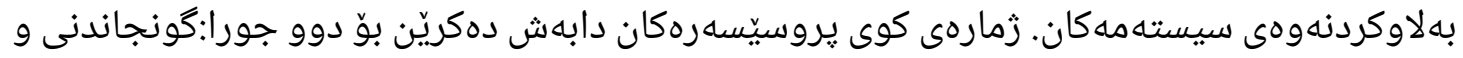

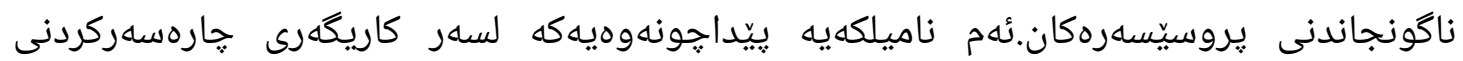

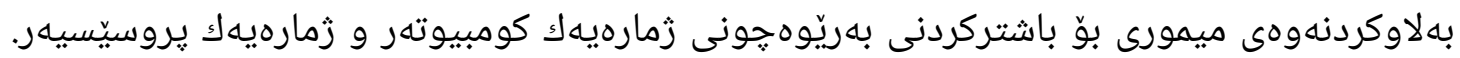

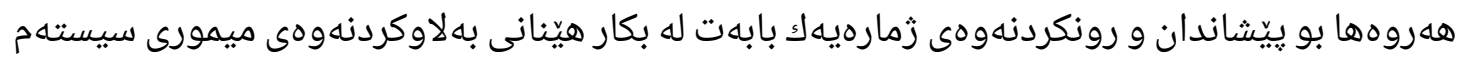

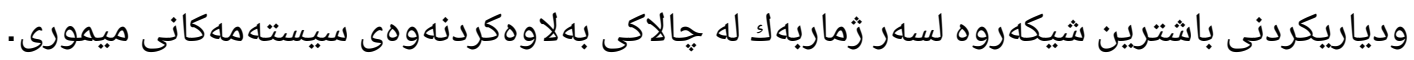

\section{تأثير نهج المعالجة المتوازية للأكرة الموزعة على تحسين أداء الأنظمة متعددة الحواسيب ومتعددة النوى: مراجعة}

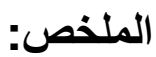

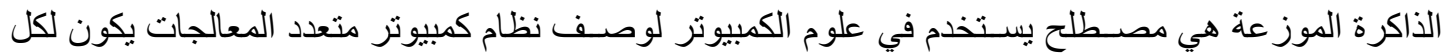

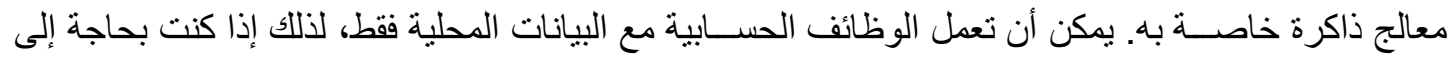

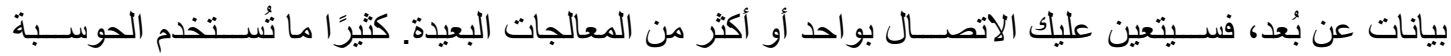

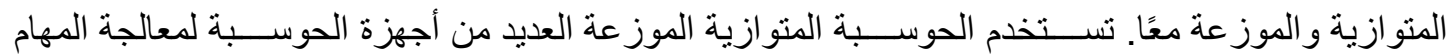




\section{QALAAI ZANISTSCIENTIFIC JOURNAL}

A Scientific Quarterly Refereed Journal Issued by Lebanese French University - Erbil, Kurdistan, Iraq

Vol. (6), No (4), Autumn 2021

بالتوازي، بينما تستخدم الحوسبة المتوازية على جهاز كمبيوتر واحد معالجات متعددة لتنفيذ المهام بالتوازي. تم

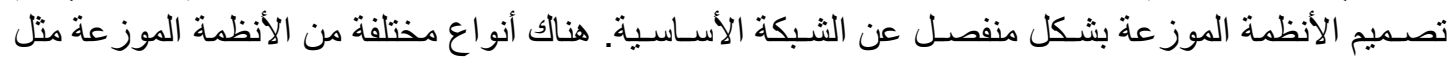
شبكات الند للند (P2P) و المجمو عات و الثبكات و أنظمة التخزين الموزعة. يمكن تصنيف المعالج متعدد النواة

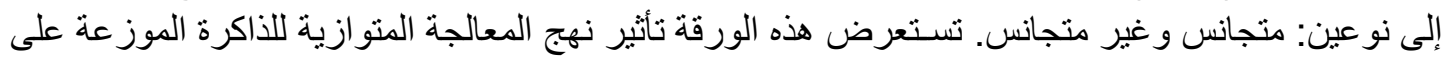

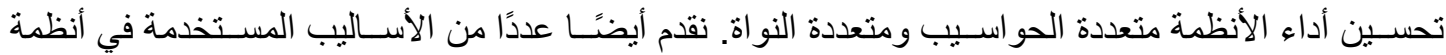

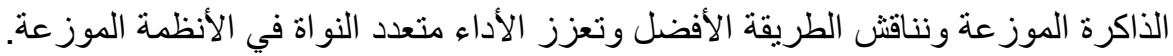

